\title{
Science projects with the Armenian Virtual Observatory (ArVO)
}

\author{
Areg M. Mickaelian \\ Byurakan Astrophysical Observatory, Byurakan 378433, Aragatzotn province, Armenia \\ email: aregmick@apaven.am
}

The main goal of the Armenian Virtual Observatory is to develop efficient methods for science projects based on the digitized famous Markarian survey (Digitized First Byurakan Survey, DFBS) and other large astronomical databases, both Armenian and international. Two groups of projects are especially productive: search for new interesting objects of definite types by low-dispersion template spectra, and optical identifications of new gamma, X-ray, IR and radio sources. The first one is based on modeling of spectra for a number of types of objects: QSOs, Seyfert galaxies, white dwarfs, subdwarfs, cataclysmic variables, planetary nebulae, late-type stars (K-M, S, carbon), etc. Each kind of object appears in the DFBS with its typical SED and spectral lines (for objects having broad lines only, like white dwarfs and subdwarfs, quasars and Seyferts, etc.), however affected also by its brightness, so that each template works for definite range of magnitudes. The search criteria define how many objects will be found for further study, and may restrict these numbers leaving with the best candidates. Optical identifications have been proven to be rather efficient for IR sources from IRAS-PSC and -FSC. Tests have been carried out for X-ray and radio sources as well. Special emphasis is put on search for bright QSOs missed by the Sloan Digital Sky Survey (SDSS), rather important for making up their complete sample, studies of the properties of the Local Universe, a comparison of their X-ray, IR, and radio properties and making up their multiwavelength SEDs, as well as for a refinement of the AGN classification. A project for search for new bright QSOs using the DFBS has been started in the region with $\delta>0^{\circ}$ and $|b|>20^{\circ}$. The Byurakan $2.6 \mathrm{~m}$ telescope is being used for the spectral identification of the candidates. The first test resulted in 145 objects found, 81 being known QSOs/Sys, and 64 new candidates (including 23 NVSS and FIRST radio sources).

A number of sub-projects have been tested concerning the asteroids search on the DFBS plates. All known bright $\left(<15^{\mathrm{m}}-16^{\mathrm{m}}\right)$ asteroids have been grouped into fast and slow ones with a division parameter, estimated as the motion of $3^{\prime \prime}$ during $20 \mathrm{~min}$ (the typical exposure time of a DFBS plate). Extraction of the spectra of asteroids found in DFBS by SkyBote has been started, and they were grouped into extended (fast asteroids) and star-like (slow asteroids). The further plans include modeling of a template spectra of asteroids by means of the star-like spectra, search for new candidate asteroids by similar spectra and comparison with DSS1/DSS2 fields for elimination of the stars, spectral analysis of the asteroid spectra to get some physical parameters, etc.

Another project is the identification of the newly found IR sources from Spitzer Space Telescope (SST). 73 unidentified sources in the Bootes region have been found and classified on the DFBS plates. All available additional data from DSS1/DSS2, other optical and multiwavelength catalogs were used to clarify the nature of these objects. 51 were found to be known objects from existing catalogs, including 1 QSO, 28 galaxies, and 22 stars. The 22 new objects were classified as five candidate QSOs, ten galaxies (four AGN candidates and six interacting systems), and seven stars (six G-M type, and one carbon). Eight of the known stars not having spectral classification, were classified too. 